\title{
Unexpected rhythm regularity in a patient with atrial fibrillation and a changing frontal plane axis over time
}

\author{
A. Zweerink ${ }^{1}$ E. Leusveld ${ }^{1}$ C. P. Allaart ${ }^{1}$ M. J. B. Kemme ${ }^{1}$
}

Published online: 26 October 2016

(C) The Author(s) 2016. This article is available at SpringerLink with Open Access.

\section{Answer}

Both ECGs show a regular, relatively narrow-complex tachycardia of $150 \mathrm{bpm}$. The first ECG shows an incomplete right bundle branch block (RBBB) pattern in the precordial leads, with the extremity leads demonstrating a left-axis deviation. In the second ECG the frontal plane axis has changed towards a right-axis deviation. Both tachycardias originate from the left bundle branch-Purkinje system causing an incomplete RBBB pattern. In the first ECG, the origin of this fascicular ventricular tachycardia (VT) is located in the posterior fascicle (left-axis deviation) and during the second ECG in the anterior fascicle (right-axis deviation) as illustrated in Fig. 1. In idiopathic fascicular VT, the most likely mechanism is reentry by an excitable gap and a zone of slow conduction [1]. Here, the most likely mechanism is triggered activity in the region of the fascicles due to digoxin intoxication. In this specific case the digoxin level was $3.4 \mu \mathrm{g} / \mathrm{l}$ (reference: max level $2.0 \mu \mathrm{g} / \mathrm{l}$ ). As digoxin inhibits the ATPase dependent sodium-potassium pump, increased intracellular calcium concentrations lead to delayed afterdepolarisations and triggered activity [2]. An important feature of the delayed afterdepolarisations is that they can be exacerbated by a shortening of the cycle length and by catecholamines, in this case by atrial fibrillation with rapid ventricular conduction. In rare cases the frontal plane axis alternates on a beat-to-beat basis, this 'bidirectional VT' indicates se-
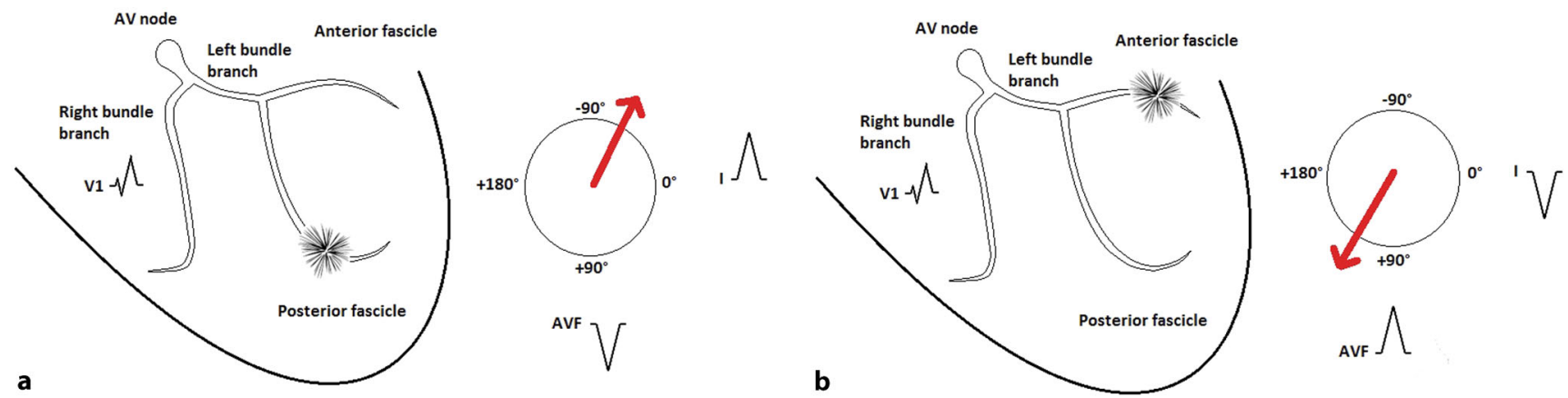

Fig. 1 Both figures illustrate a fascicular VT originating from the left bundle branch-Purkinje system, with a focus in the posterior fascicle (a) and in the anterior fascicle (b), respectively. In both cases the right ventricle is the last to be activated, causing an incomplete RBBB pattern in V1. Impulse formation from the posterior fascicle results in a left-axis deviation (a), whereas impulse formation from the anterior fascicle results in a right-axis deviation $(\mathbf{b})$

A. Zweerink

a.zweerink@vumc.nl

1 Department of Cardiology, VU University Medical Center, Amsterdam, The Netherlands 


\section{Advertisement placed here.}

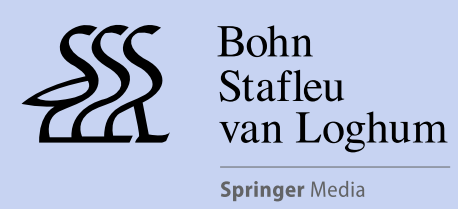

Houten 2017 


\section{Advertisement placed here.}

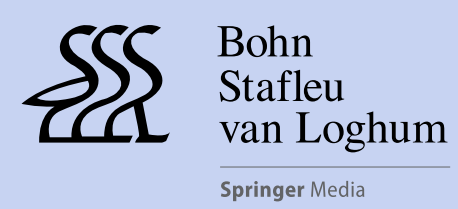

Houten 2017 
vere digoxin intoxication and is considered life-threatening [3]. Due to its automatic nature, electrical cardioversion is usually not successful. Digoxin-induced fascicular VT is generally responsive to digoxin-specific antibodies with which this patient was successfully treated.

Open Access This article is distributed under the terms of the Creative Commons Attribution 4.0 International License (http:// creativecommons.org/licenses/by/4.0/), which permits unrestricted use, distribution, and reproduction in any medium, provided you give appropriate credit to the original author(s) and the source, provide a link to the Creative Commons license, and indicate if changes were made.

\section{References}

1. Nogami A, Naito S, Tada H, et al. Demonstration of diastolic and presystolic Purkinje potentials as critical potentials in a macroreentry circuit of verapamil-sensitive idiopathic left ventricular tachycardia. J Am Coll Cardiol. 2000;36:811-23.

2. Rosen MR. Cellular electrophysiology of digitalis toxicity. J Am Coll Cardiol. 1985;5(Suppl A):22A-34A.

3. Baher AA, Uy M, Xie F, Garfinkel A, Qu Z, Weiss JN. Bidirectional ventricular tachycardia: ping pong in the His-Purkinje system. Heart Rhythm. 2011;8:599-605. 